\title{
RB1CC1 activates the $p 16$ promoter through the interaction with hSNF5
}

\author{
YASUKO OCHI $^{1,4}$, TOKUHIRO CHANO ${ }^{1}$, KAICHIRO IKEBUCHI $^{1,4}$, HIROKAZU INOUE $^{2}$, TAKAHIRO ISONO $^{3}$ \\ AKIHITO ARAI $^{1,4}$, HITOSUKE TAMENO ${ }^{1,4}$, TAKETOSHI SHIMADA ${ }^{4}$, YASUO HISA ${ }^{4}$ and HIDETOSHI OKABE ${ }^{1}$ \\ Departments of ${ }^{1}$ Clinical Laboratory Medicine, ${ }^{2}$ Microbiology and ${ }^{3}$ Central Research Laboratory, Shiga University \\ of Medical Science, Tsukinowa-cho, Seta, Otsu, Shiga 520-2192; ${ }^{4}$ Department of Otolaryngology-Head and Neck \\ Surgery, Kyoto Prefectural University of Medicine, Kajiicho 465, Kamigyo-ku, Kyoto 602-8566, Japan
}

Received January 12, 2011; Accepted March 10, 2011

DOI: $10.3892 /$ or.2011.1329

\begin{abstract}
RB1-inducible coiled-coil 1 (RB1CC1, also known as FIP200) is involved in dephosphorylation and increase of retinoblastoma tumor suppressor protein (RB1), but the RB1CC1 molecular mechanism in the dephosphorylation of $\mathrm{RB} 1$ is not fully understood. We determined that RB1CC1 activates the expression of p16 (also called INK4a/CDKN2a) through the activation of its promoter, using chromatin immunoprecipitation (ChIP) and pl6 promoter-luciferase reporter assays. In addition, RB1CC1 essentially requires binding with hSNF5 (also known as BAF47/INI1, a chromatin-remodeling factor) to activate the 16 promoter, in order to enhance the RB1 pathway and acts as a tumor suppressor. Evaluation of the RB1CC1 mechanism of action is expected to provide useful information for clinical practice and future therapeutic strategies in human cancers.
\end{abstract}

\section{Introduction}

INK4a/CDKN2a is generally known as p16, a tumor suppressor protein that functions as an inhibitor of CDK4 and CDK6, which initiate phosphorylation of retinoblastoma tumor suppressor protein, RB1. p16 induces G1 cell cycle arrest, and plays a physiological role in the implementation of irreversible growth arrest (1). Thus, p16 plays a key role in cellular senescence in human cells, and is important in resistance to oncogenic transformation (1). It is known that pl6 transcription is activated by hSNF5 (2-4) and Ets1/2 $(5,6)$, and is inhibited by Id1 and Bmi-1 (7-9). Genetic mutation and deletion of these p16 regulators have not been widely reported, while hSNF5 is mutated with loss of functionality in malignant rhabdoid tumor (MRT), a rare and extremely aggressive childhood cancer. Indeed, the functional relation-

Correspondence to: Dr Tokuhiro Chano, Department of Clinical Laboratory Medicine, Shiga University of Medical Science, Tsukinowa-cho, Seta, Otsu, Shiga 520-2192, Japan

E-mail: chano@belle.shiga-med.ac.jp

Key words: RB1-inducible coiled-coil 1, p16, hSNF5, promoter, cancer ship between p16 and RB is frequently deregulated in many types of human cancer. However, the mutations and deletions in $\mathrm{p} 16, \mathrm{RB}$ and upstream regulators of this pathway are not frequent (10). There could presumably be other factors than the p16 and $\mathrm{RB}$ that regulate the pathway relating to progression or prevention of tumorigenesis.

RB1-inducible coiled-coil 1 [RB1CC1: the symbol referred to here and approved by the Human Genome Organization (HUGO) Gene Nomenclature Committee; also known as FIP200] was identified as an RB1 regulator. In our previous report, we described that $\mathrm{RB} 1 \mathrm{CC} 1$ activates the $\mathrm{RB} 1$ promoter through the 201 bp upstream GC-rich region (11). We have also reported that RB1CC1 forms a complex with hSNF5 and/or p53, and coordinates with it to activate the p16 promoter (12).

In the present study, we initially performed an immunohistochemical analysis, and noted that nuclear RB1CC1 expression was significantly correlated with p16 expression. In addition, the labeling indices of hSNF5 were constantly high $(\sim 80 \%)$ in all cases of breast cancer, and there was no correlation between hSNF5 and p16. We hypothesized that $\mathrm{RB} 1 \mathrm{CC} 1$ regulated the quantitative activation of the $p 16$ promoter. Therefore, we analyzed the mechanism of how RB1CC1 regulated pl6 transcription enhancing its amount. Here, we discovered that RB1CC1 controlled the transcription level of p16 in the presence of hSNF5, and that RB1CC1 was an upstream regulator of $p 16$ transcription in many different types of cancer, except for MRT, an extremely rare malignancy.

\section{Materials and methods}

Antibodies. Rabbit antisera against RB1CC1 (aa. 25-271 as epitope) were generated as previously reported (13), and the purified $\mathrm{IgG}$ fractions were used in the experiments. Anti-p16 (G175-405) and anti-BAF47 (25) were purchased from BD Bioscience (NJ, USA). Anti-HA (3F10) and anti-Flag (1E6) were from Roche (CT, USA) and Wako (Japan, Osaka), respectively.

Histology and immunohistochemistry. To evaluate the correlation between RB1CC1, p16 and hSNF5 in human breast cancer 
tissue, surgical specimens from 59 cases were embedded in paraffin after overnight fixation in $10 \%$ buffered formalin, and processed for a conventional histopathological diagnosis according to the World Health Organization (WHO) guidelines. Then, serially sliced $4-\mu \mathrm{m}$ sections from a specimen of each case were deparaffinized and incubated overnight at $4^{\circ} \mathrm{C}$ with primary antibodies to RB1CC1, p16 and hSNF5 after immersion in $0.3 \% \mathrm{H}_{2} \mathrm{O}_{2}$ and autoclaving at $120^{\circ} \mathrm{C}$ for $1 \mathrm{~min}$. The sections were rinsed with $1 \mathrm{X}$ PBS and incubated with the secondary antibody (Simple Stain MAX-PO; Nichirei, Japan) at room temperature for $1 \mathrm{~h}$. The sections were then stained with 3,3'-diaminobenzidine tetrahydrochloride (DAB) and counterstained with hematoxylin.

Microscopic evaluation and statistical analysis of correlation between RB1CC1 staining and the labeling index of p16 or hSNF5. The extent of RB1CC1 expression was classified into three categories: I, negative stain in both cytoplasm and nuclei; II, positive stain only in cytoplasm and negative in nuclei; III, positive stain in nuclei and/or cytoplasm. For p16 and hSNF5, a nuclear staining reaction that was brownish in color was labeled as positive. The p16 and hSNF5 labeling indices were determined as follows: positive tumor cells/total tumor cells x100. One-way factorial ANOVA and multiple comparison tests accompanied by Scheffe's significance were used to evaluate the relationships between RB1CC1 grade and p16 labeling index. Spearman's rank correlation coefficient was used to evaluate the correlations between hSNF5 and p16 labeling indices.

Cell culture. HeLa, TTC642, H1299 and HEK293 cells were purchased from either American Type Culture Collection (MD, USA) or Japanese Collection of Research Bioresources (Osaka, Japan), and cultured in Dulbecco's modified Eagle's medium (DMEM) or RPMI-1640 containing $10 \%$ fetal bovine serum (FBS). All cell culture media were supplemented with penicillin (50 units $/ \mathrm{ml})$ and streptomycin $(50 \mathrm{mg} / \mathrm{ml})$.

Plasmid DNA and gene transfer. The mammalian expression vector for RB1CC1 (RB1CC1wt: pcDNA-RB1CC1) was generated as reported previously (14). The luciferase plasmid, including the $p 16$ ( $p 16$ wild-type: $\mathrm{p} 16 \mathrm{wt}$ ), $R B 1, \mathrm{p} 21$ or TP53AIP1 promoter, was constructed as reported previously (12). p16 mutant plasmids (A, B, C, AB and BC) were generated by a combination of PCR-based manipulations with appropriate external primers at the positions described below and restriction enzyme digestion. Referring to Fig. 2A, the p16-mutants $\mathrm{A}, \mathrm{B}, \mathrm{C}, \mathrm{AB}$ and $\mathrm{BC}$ contained -870 to -624 , -624 to $-315,-326$ to $-22,-870$ to -315 and -624 to -22 , respectively (the initiation ATG was defined as +1 ). The plasmids of p16 promoter (-Ebox1, -GT, -SpW, -sGC, -iGC, -Sp1, -Sp2, -Ebox2, -NFאB, -GATA, -Ets1,GATA, -Ets1, -Ets3, -Ets2,Ets3, -Ets1,Ets3 and -Ets1,Ets2,Ets3) were generated. Referring to Fig. 2B, p16mut-Ebox1 (5'-GTACAGGaaATTTCG-3'), -GT (5'-TTCTCGaaaGGGCTC-3'), -SpW (5'-ACGGGCaaaaaCG GATTT-3'), -sGC (5'-AACGGGaaaGGGGCG-3'), -iGC (5'-GCGGGGaaaGATTTC-3'), -Sp1 (5'-CTGGCAG aaaGG GGAGC-3'), -Sp2 (5'-CGGAGGaaaGTGTGG-3'), and -Ebox2 (5'-GGGCAGGaaGGGGAGG-3') were mutated from the wild-type pl6 promoter sequence (5'-GTACAGGTATTT
CG3'), (5'-TTCTCGGTGGGGCTC-3'), (5'-ACGGGGCG GGGGCGGATTT-3'), (5'-AACGGGGCGGGGGCG-3'), (5'-GCGGGGGCGGATTTC-3'), (5'-CTGGCAGGCG GGGGAGC-3'), (5'-CGGAGGGCGGTGTGG-3'), and (5'-GGGCAGGTGGGGAGC-3'), respectively. Referring to Fig. 2C, p16mut-NFkB (5'-CCCCGGacGAGAgCAACCT-3'), -GATA (5'-CTGGAA AaATgCCGCGGT-3'), -Ets1 (5'-GCTaCTTaaGGCTGGT-3'), -Ets2 (5'-GCTCTTaaGCC AGCA-3'), and -Ets3 (5'-AGCACCccAttAAGAAAGA-3') were mutated from the wild-type $p 16$ promoter sequence, (5'-CCCCGGGGGAGACCCAACCT-3'), (5'-CTGGAA AGATACCGCGGT-3'), (5'-GCTGCTTCCGGCTGGT-3'), (5'-GCTCTTCCGCCAGCA-3'), and (5'-AGCACCGG AGGAAGAAAGA-3'), respectively. p16mut-Ets1,GATA, -Ets2,Ets3, -Ets1,Ets3 and -Ets1,Ets2,Ets3 contained (Ets1 and GATA), (Ets2 and Ets3), (Ets1 and Ets3) and (Ets1, Ets2 and Ets3) mutant sequences, respectively. Referring to Fig. 5A, flag-tagged mutants of RB1CC1; dCCB and dLZ, were generated by deleting the amino acids of 1078-1363 and 1357-1594 for RB1CC1 wild-type, respectively. All plasmid constructs were made by PrimeSTAR ${ }^{\mathrm{TM}}$ Mutagenesis Basal Kit (Takara, Japan) according to the manufacturer's instructions, and confirmed by DNA sequencing. Transfection preceded using FuGene HD (Roche) or Lipofectamine 2000 (Invitrogen) according to the supplier's recommendations.

Luciferase-reporter assay. The p16 wild-type- or mutant-, RB1-, p21- or TP53AIP1- luciferase reporter plasmid was co-transfected using FuGene HD (Roche) with empty vector (pcDNA) and/or RB1CC1-expression vector (RB1CC1wt or RB1CC1mut) into the HeLa cells one day after seeding into $35-\mathrm{mm}$-well plates at the concentration of $4 \times 10^{4}$ cells. Twenty-four hours later, luciferase activity was analyzed using a luciferase assay kit (Toyo Inc., Japan) and luminometer (EG\&G Berthold Lumat LB9507). $\beta$-gal activity was evaluated as an internal control for transfection efficiency using Aurora ${ }^{\mathrm{TM}}$ kit (ICN Biomedicals, CA, USA).

Chromatin immunoprecipitation (ChIP) assay. ChIP assay of the endogenous $p 16$ promoter regions in HeLa or TTC642 cells was done by the ChIP-IT ${ }^{\mathrm{TM}}$ control and ChIP-IT ${ }^{\mathrm{TM}}$ Express kits (Active Motif, CA, USA) according to the manufacturer's instructions using anti-RB1CC1 IgG, antihSNF5 IgG and control IgG (Active Motif). The amount of co-immunoprecipitated DNA was evaluated by the quantitative polymerase chain reaction (qPCR). The amplified region of pl6 promoter was from -711 to -529 (the initiation ATG was defined as +1 ). The sequences of the PCR primers were 5'-AGGAATCCTTTGAACTAGGG-3' and 5'-GGGAAAGTATGGCTTCTTCG-3'. The PCR conditions were multiple cycles of 3 steps $\left(98^{\circ} \mathrm{C}, 10 \mathrm{sec}\right.$ at $58^{\circ} \mathrm{C}, 30 \mathrm{sec}$ at $\left.68^{\circ} \mathrm{C}, 30 \mathrm{sec}\right)$.

Immunoprecipitation and Western blotting. Before immunoprecipitation, cells were lysed in 1X TNE buffer $(20 \mathrm{mM}$ Tris- $\mathrm{HCl}$ containing $150 \mathrm{mM} \mathrm{NaCl}, 5 \mathrm{mM}$ EDTA, $1 \%$ NP-40 and $1 \mathrm{mM} \mathrm{Na} \mathrm{VO}_{4}$ and a mixture of protease inhibitors). After cleaning lysed material by centrifugation at $15,000 \mathrm{rpm}$ for $10 \mathrm{~min}$, the supernatant was rotary-incubated 
A

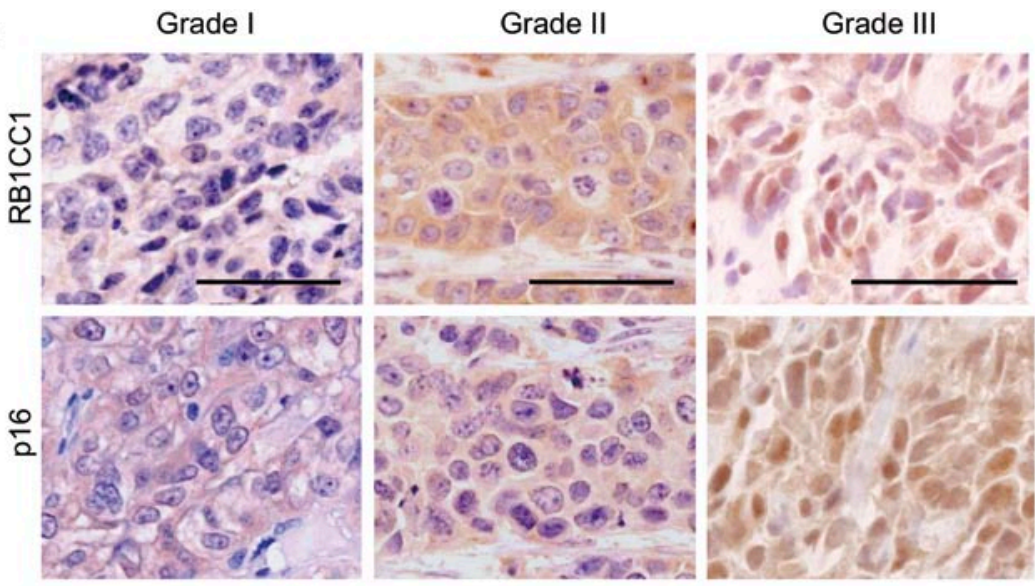

B

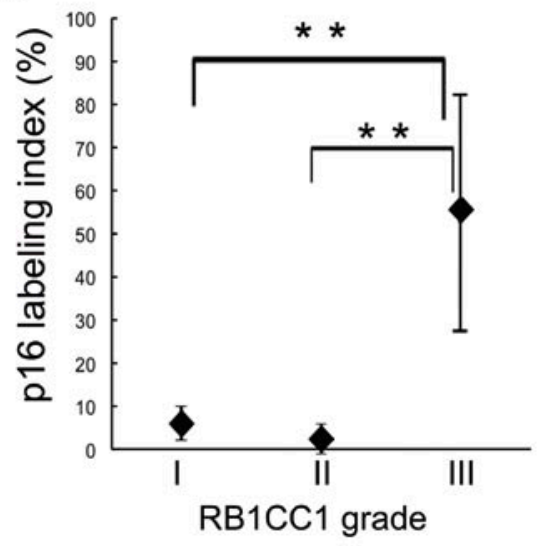

C

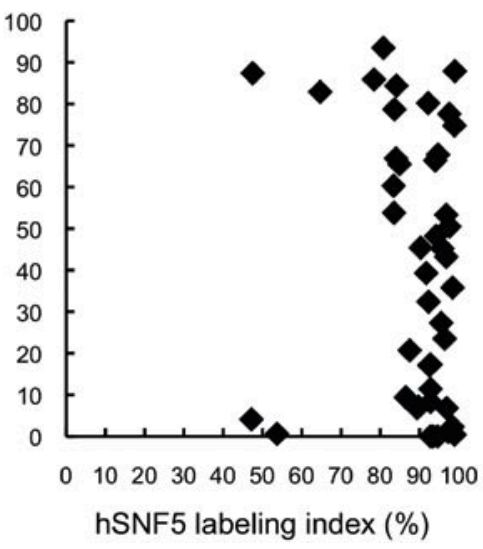

Figure 1. RB1CC1 regulates p16 expression in cancer tissues. (A) Representative cases of immunohistochemical RB1CC1 grades of expression are demonstrated in the upper row, and p16 expressions in each serial section are shown in the lower row. Grade I is defined as negative staining in both cytoplasm and nuclei; II, positive staining only in cytoplasm and negative in nuclei; III, positive staining in nuclei and/or cytoplasm. p16 expression is remarkable in RB1CC1 grade III case. Each scale bar represents $50 \mu \mathrm{m}$. (B) The relationship between the expressional grade of RB1CC1 and p16 labeling index in breast cancer. p16 labeling indices (mean \pm SD) were $5.94 \pm 4.11 \%, 2.37 \pm 3.44 \%$ and $55.60 \pm 29.56 \%$ in grade I (8 cases), II (6 cases), and III (45 cases) RB1CC1 expression categories, respectively. p16 labeling index is significantly high in the case with nuclear RB1CC1 expression (grade III). One-way factorial ANOVA and multiple comparison tests accompanied by Scheffe's significance were used to evaluate the relationship $\left({ }^{* *} \mathrm{p}<0.01\right)$. (C) The relationship between hSNF5 and p16 labeling indices in breast cancer. No statistical significance was detected in the relationship by Spearman's rank correlation coefficient.

with anti-HA (Roche) immobilized beads for $2 \mathrm{~h}$ at $4^{\circ} \mathrm{C}$. The beads were washed five times with $1 \mathrm{X}$ TNE buffer and boiled in SDS sample buffer. Proteins resolved by SDS-PAGE were transferred to polyvinylidene difluoride (PVDF) membranes and immunoblotted with the indicated antibodies.

\section{Results}

RB1CC1 and p16 expressions correlate in cancer tissues. $\mathrm{RB} 1 \mathrm{CC} 1$ and p16 were immunohistochemically analyzed in 59 cases of breast cancers. Nuclear RB1CC1 expression correlated with high p16 expression (Fig. 1A). p16 labeling indices (mean \pm standard deviation) were $5.94 \pm 4.11 \%, 2.37 \pm 3.44 \%$ and $55.60 \pm 29.56 \%$ in grade I ( 8 cases), II (6 cases), and III (45 cases) RB1CC1 expression categories, respectively. The p16 labeling index was significantly high in grade III cases of RB1CC1 (One-way factorial ANOVA and multiple comparison tests accompanied by Scheffe's significance, $\mathrm{p}<0.01$; Fig. 1B). These results suggested that RB1CC1 nuclear expression correlated with the p16 expression level.
It has been reported that hSNF5 largely contributes to p16 expression (2-4). To evaluate the contribution of hSNF5 to p16 expression in vivo, we immunohistochemically analyzed the labeling indices of hSNF5 and p16 in similar cases of breast cancers. The labeling indices of hSNF5 were $>80 \%$ in most of the breast cancer cases, and there was no correlation between hSNF5 and p16 labeling indices (Fig. 1C).

RB1CC1 enhances the activity of p16 promoter. In order to identify the critical region for $\mathrm{RB} 1 \mathrm{CC} 1$ to activate the $p 16$ promoter, the luciferase reporter assay was used. Wild-type (p16wt) and the various deletion mutants (A, B, C, AB and BC) of the $p 16$ promoter reporter plasmid were co-transfected into HeLa cells with the RB1CC1-expression vector (RB1CC1) or empty (pcDNA) plasmid (Fig. 2). While the luciferase activity of the p16 mutant A (-870 to $-624 \mathrm{bp})$ was approximately equal to that co-transfected with pcDNA, the promoters, which contained the region of $\mathrm{B}$ ( -624 to $-315 \mathrm{bp})$ and/or $\mathrm{C}$ (-326 to $-22 \mathrm{bp}$ ), were $\sim 3-5$-fold enhanced by RB1CC1. Importantly, the promoter containing both regions ( $\mathrm{BC},-624$ to $-22 \mathrm{bp}$ ) 
A

A B $C$

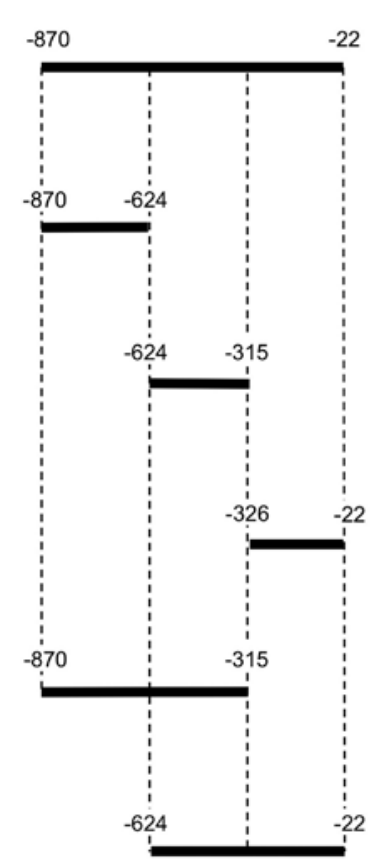

B

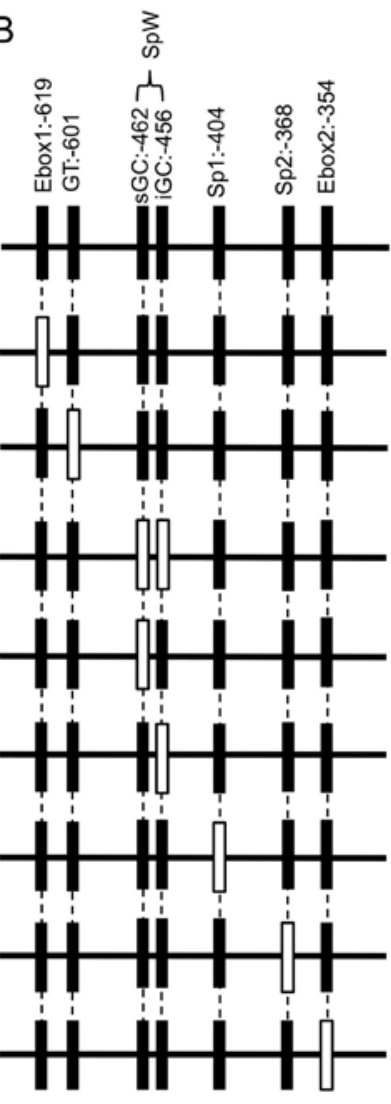

Luciferase activity ( $\%$ of control)
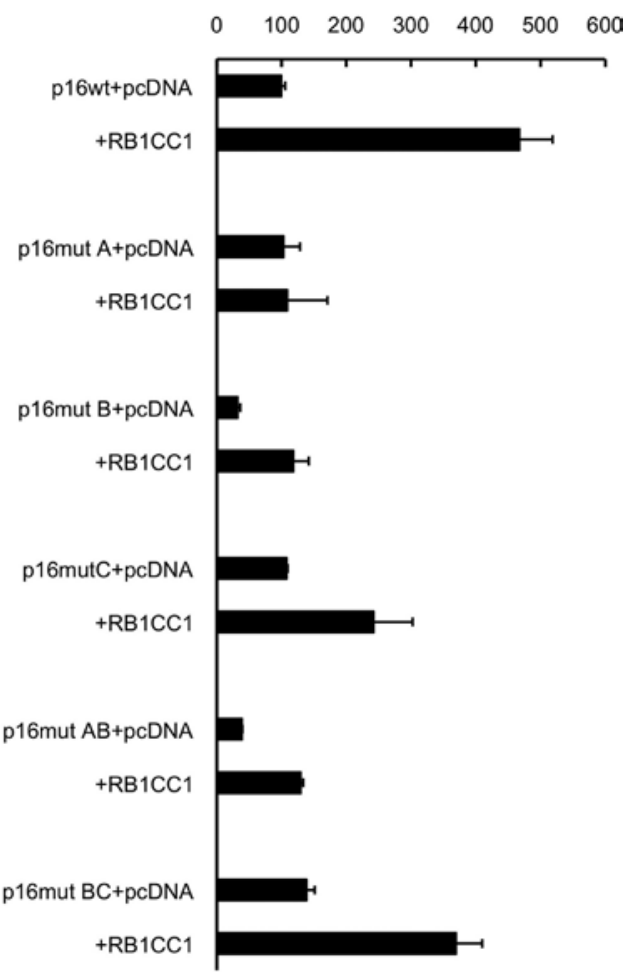

Luciferase activity (\% of control)

0100200300400500600700800900

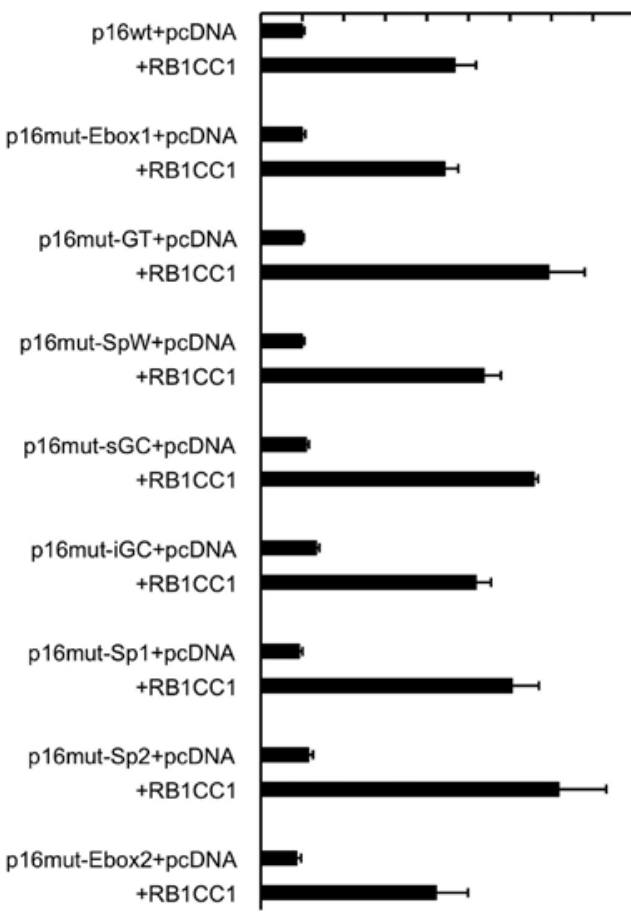

Figure 2. RB1CC1 enhances $p 16$ promoter activity. (A) Schematic representation (left) of p16wt and five kinds of reporter plasmids containing $p 16$ promoter deletion-mutants (p16mut A, B, C, AB and BC) used in luciferase assays, corresponding to the graph on the right. (B) Schematic representation (left) of $p 16 w t$ and eight kinds of reporter plasmids containing point-mutations of p16 promoter (p16mut-Ebox1, -GT, -SpW, -sGC, -iGC, -Sp1, -Sp2 and -Ebox2) used in luciferase assays, corresponding to the graph on the right.

was additively stimulated by RB1CC1, and reached similar levels to that of the wild-type promoter (p16wt). These results suggested that $\mathrm{RB} 1 \mathrm{CC} 1$ required the limited domain (-624 to $-22 \mathrm{bp})$ of the 16 promoter to activate the promoter in a dose 
C

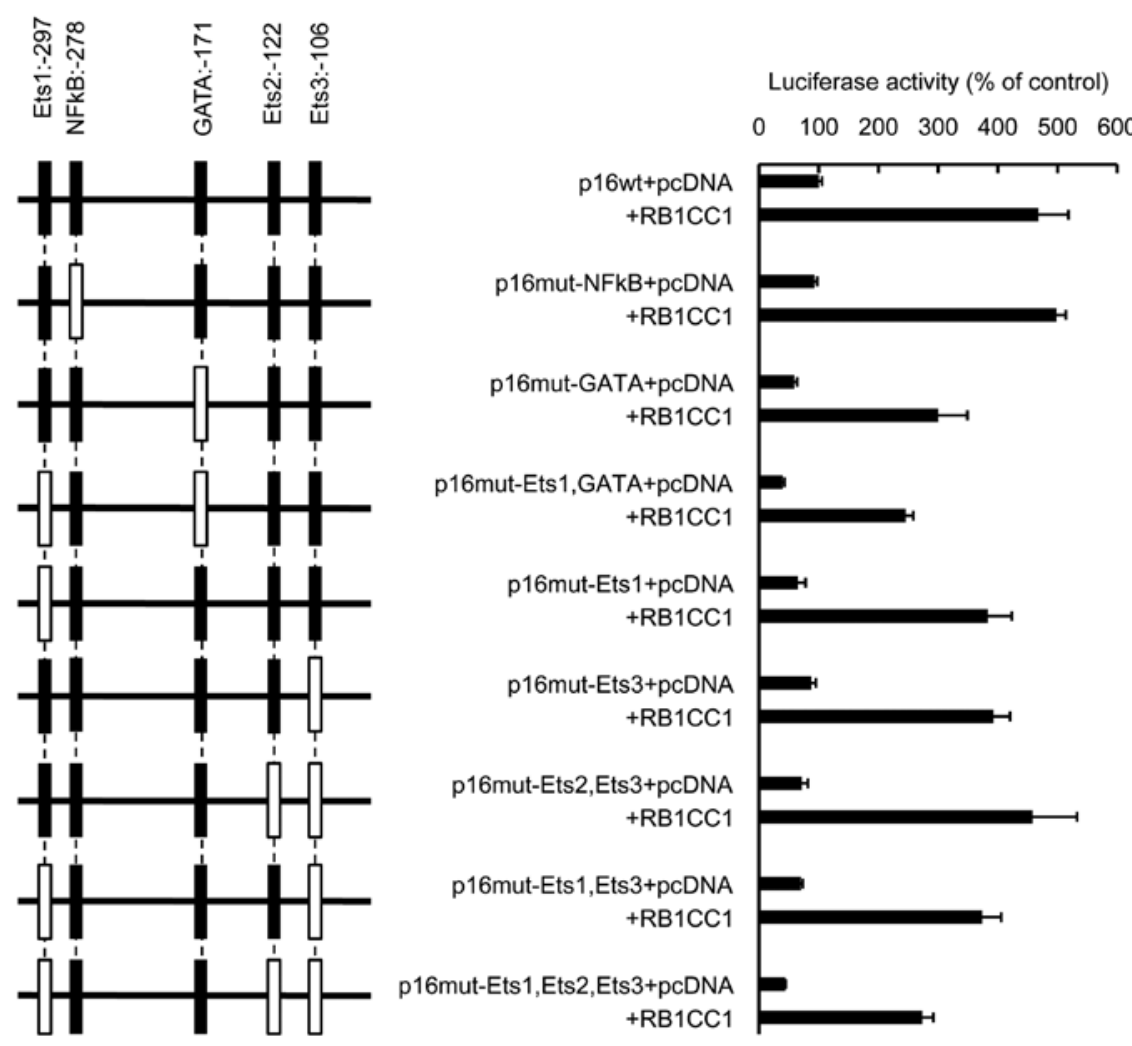

Figure 2. (C) Schematic representation (left) of p16wt and eight kinds of reporter plasmids containing point-mutations of p16 promoter (p16mut-NFkB, -GATA, -Ets1GATA, -Ets1, -Ets3, -Ets2Ets3, -Ets1Ets3 and -Ets1Ets2Ets3) used in luciferase-reporter assays, corresponding to the right graph. The translation start site is determined as +1 . All the luciferase values were calculated as the relative fold induction versus $p 16 w t$ co-transfected with pcDNA. The values indicated the means \pm standard errors from quadruplicate experiments in HeLa cells.

(DNA-length) -dependent manner. Additionally, to identify the critical narrow domain of the $p 16$ promoter activated by RB1CC1, we modified the $p 16$ wild-type reporter plasmid and screened each activity co-transfected with RB1CC1. We made 8 (p16mut-Ebox1, -GT, -SpW, -sGC, -iGC, -Sp1, -Sp2, -Ebox2) and 8 (p16mut-NFkB, -GATA, -Ets1GATA, -Ets1, -Ets3, -Ets2Ets3, -Ets1Ets3 and -Ets1Ets2Ets3) kinds of the promoter mutants in the regions of B (-624 to -315 bp) and C (-326 to $-22 \mathrm{bp})$, respectively. The p16 promoter activity was maintained irrespective of any other mutations (Fig. 2B and C). Altogether, while the critical narrow domain of $p 16$ promoter was not required for the activation caused by RB1CC1 expression, the region BC (-624 to $-22 \mathrm{bp})$ was required in a DNA-length-dependent manner.

RBICC1 requires $h S N F 5$ for binding to the p16 promoter. To examine whether RB1CC1 binds to the $p 16$ promoter, the ChIP experiments were performed using HeLa and TTC642 (hSNF5-/-) cells. Immunoprecipitated DNA fragments were amplified by PCR using the primer pairs specific to the pl6 promoter (Fig. 3). In HeLa cells, the immunoprecipitates containing a 16 promoter fragment were significantly enriched by antibodies against RB1CC1 and hSNF5, in contrast to precipitates of normal IgG control. In TTC642 cells, which are genetically null for hSNF5, antibody against RB1CC1 failed to enrich the immunoprecipitates of p16 promoter fragments. Therefore, we considered that RB1CC1 binds to the -711 to $-529 \mathrm{bp}$ region of the $p 16$ promoter, and that the binding essentially requires hSNF5 to activate the $p 16$ promoter. RB1CC1 could not interact with the 16 promoter in the hSNF5-null cells such as TTC642.

RB1CC1 requires $h S N F 5$ to activate the p16 promoter. Recently, we have identified that $\mathrm{RB} 1 \mathrm{CC} 1$ requires the involvement with hSNF5 and/or p53 to enhance the RB1 pathway (12). To evaluate the requirement of hSNF5 and p53 to activate p16, we utilized HeLa, TTC642 (hSNF5-/-) and H1299 (p53-/-) cells. Luciferase plasmid containing p16 promoter was transfected into these cells together with pcDNA or RB1CC1 (Fig. 4). RB1CC1 could not activate $p 16$ promoter adequately in TTC642 and H1299 cells, while the activation increased by about 7-fold in HeLa cells. The data suggested that RB1CC1 required the involvement of hSNF5 and p53 to activate the $p 16$ promoter. Similarly, each luciferase-reporter containing RB1, p21 or TP53AIP1 was applied into these cells, and we validated the molecular requirement of hSNF5 or $\mathrm{p} 53$ for the promoter activation caused by RB1CC1. RB1CC1 could activate neither the $R B 1$ promoter in TTC642 cells, nor the $p 21$ or TP53AIP1 promoters in $\mathrm{H} 1299$ cells. These results suggested that RB1CC1 required hSNF5 to activate the $R B 1$ promoter, and $\mathrm{p} 53$ to enhance the promoters of $p 21$ and TP53AIP1 (Fig. 4). 

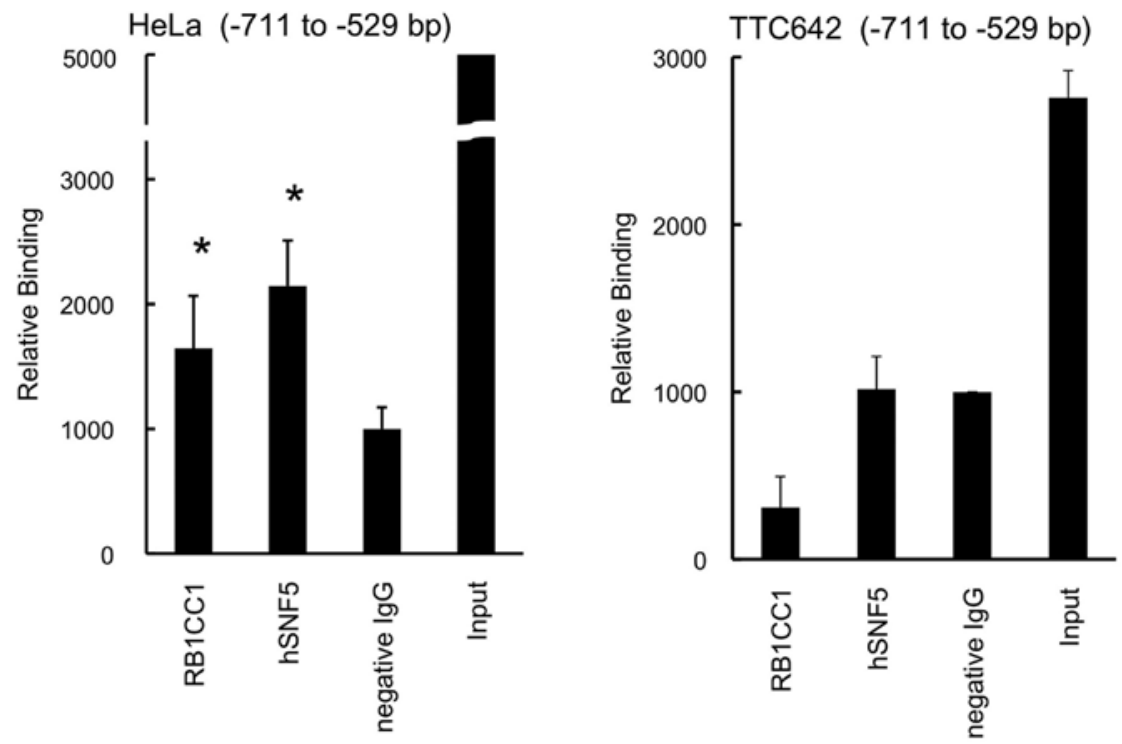

Figure 3. RB1CC1 requires hSNF5 for the binding to the p16 promoter. The ChIP PCR analysis was performed in HeLa and TTC642 (hSNF5-/-) cells using anti-RB1CC1 and anti-hSNF5 antibodies. The immunoprecipitated DNA fragments were amplified by PCR using the primer-pairs specific to $p 16$ promoter $(-711$ to $-529 \mathrm{bp})$. The values indicated the means \pm standard errors from more than quadruplicate experiments. Student's t-test; asterisks ( $\left.{ }^{\mathrm{p}}<<0.05\right)$ indicate statistically significant differences with negative IgG.

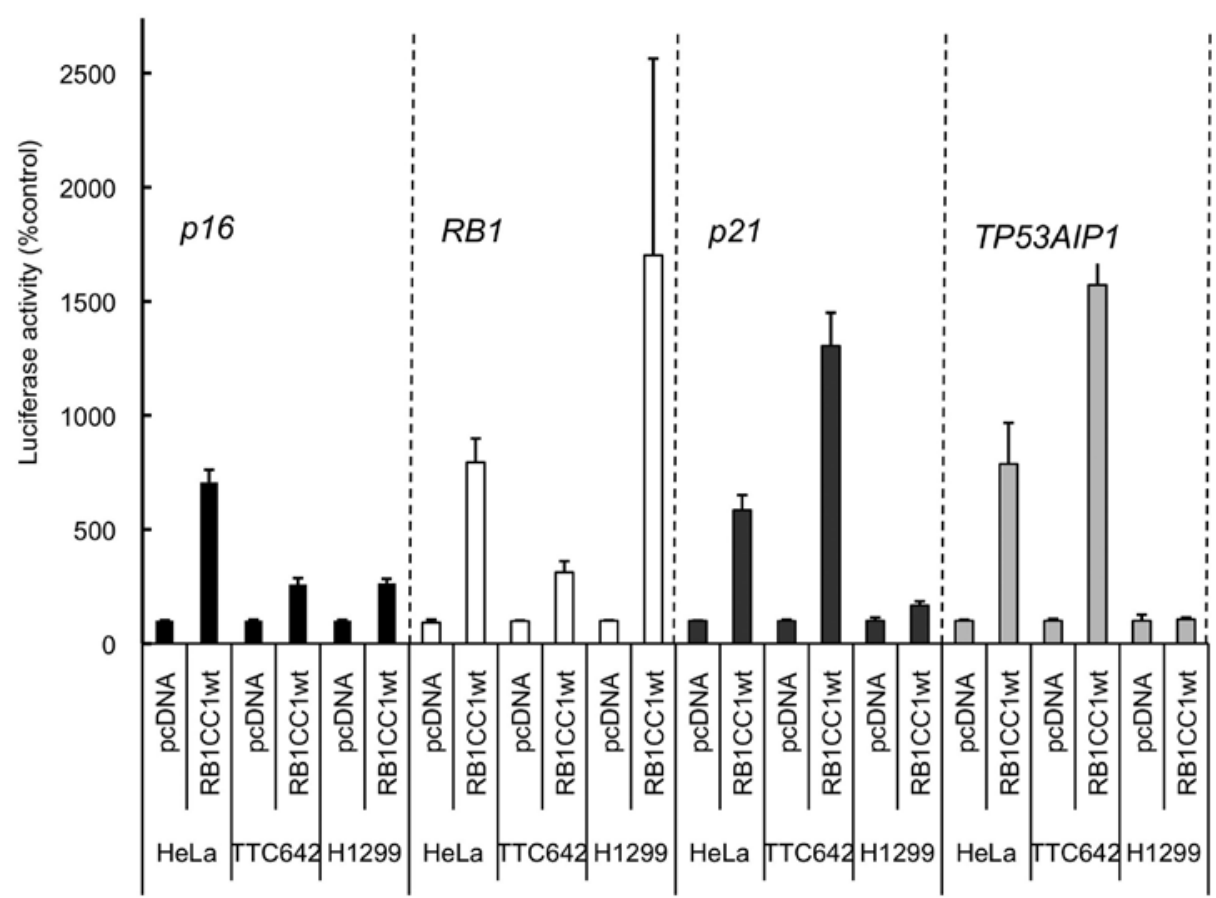

Figure 4. RB1CC1 requires the involvement with hSNF5 to activate the $p 16$ promoter. RB1CC1 also requires hSNF5 for $R B 1$ promoter activation, and needs p53 for the activations of $p 21$ and TP53AIP1. Luciferase plasmid containing RB1, p16, p21 or TP53AIP promoter was transfected into HeLa, TTC642 (hSNF-/-) or H1299 (p53-/-) cells together with RB1CC1. Luciferase activity was calculated as the relative fold induction of each versus the value of co-transfection with pcDNA. The values indicate the means \pm standard errors from quadruplicate experiments.

hSNF5 binds to C-terminus of RB1CCl, which is largely required to activate the p16 promoter. To confirm that RB1CC1 requires hSNF5 to activate the $p 16$ promoter, the binding between RB1CC1 and hSNF5 was evaluated, and it was additionally analyzed whether an RB1CC1 mutant that was unable to bind to hSNF5 could activate the $p 16$ promoter. We generated RB1CC1 deletion-mutants (dCCB: aa1078-1363 deleted; and dLZ: aa1357-1594 deleted) (Fig. 5A). HA-tagged
hSNF5 was cotransfected with the plasmids encoding flagtagged RB1CC1 wild-type or mutants in HEK293 cells. In this co-immunoprecipitation assay, hSNF5 could not bind the dLZ mutant, which had lost the C-terminal region of RB1CC1, but could bind the dCCB mutant and RB1CClwt (Fig. 3B). In addition, $p 16$ promoter activity caused by RB1CC1wt or the mutants was validated using the luciferase assay. The RB1CC1 dLZ mutant that was unable to bind to hSNF5, could 


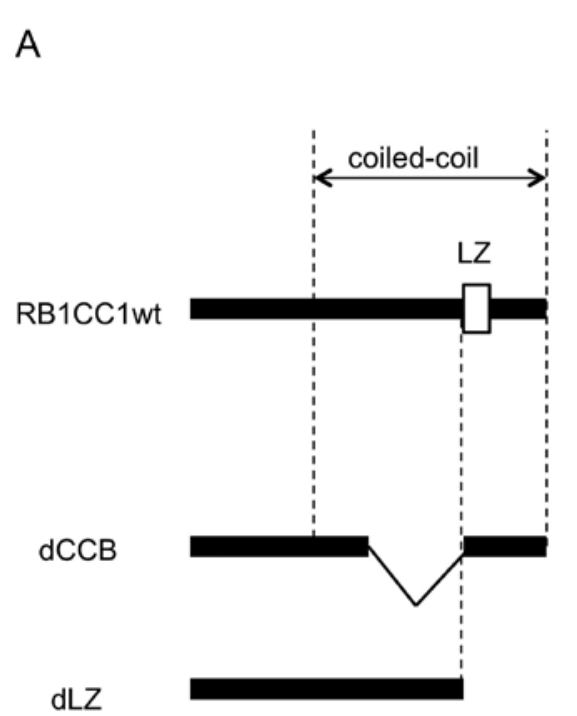

B

FlagRB1CC1: wt dCCB dLZ pcDNA hSNF5-HA: Full Full Full Full
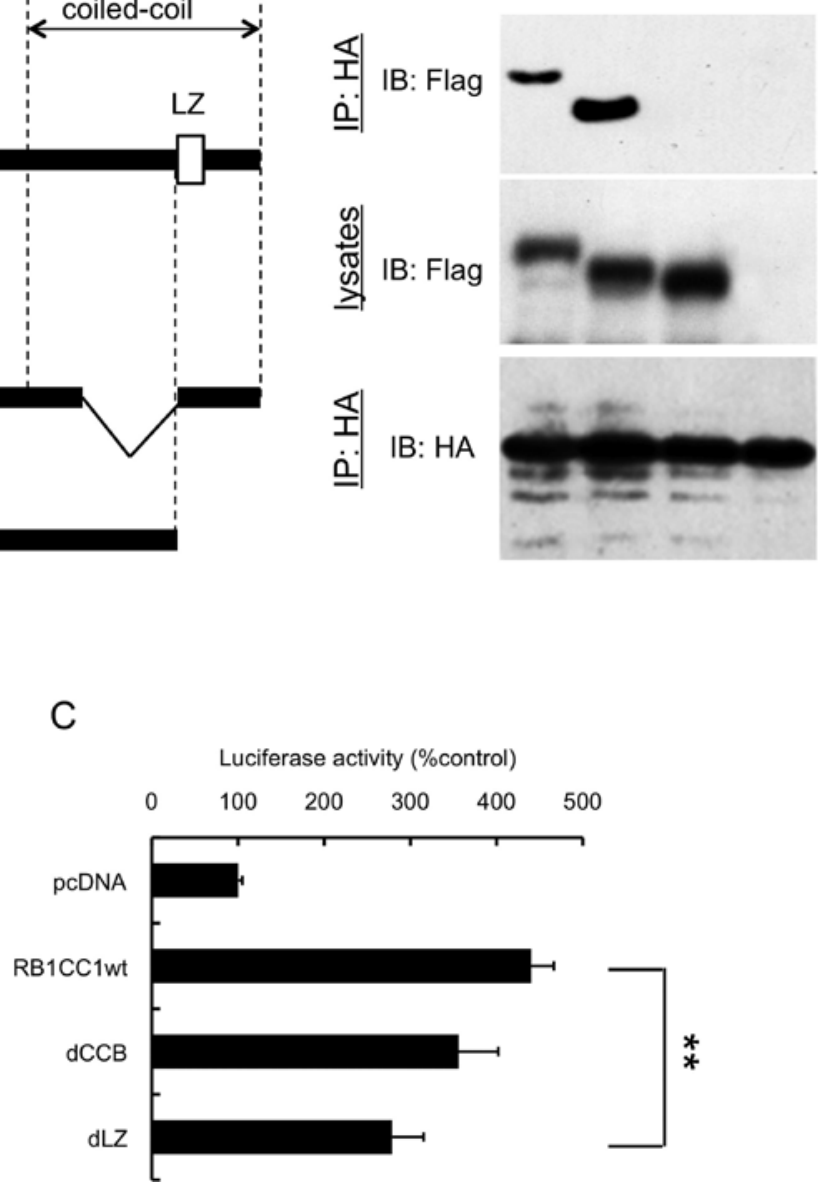

Figure 5. hSNF5 binds to the C-terminus of RB1CC1, which is essentially required for p16 promoter activation. (A) Schematic representation (left) of RB1CC1wt and two kinds of RB1CC1 deletion-mutants (dCCB; aa1078-1363 deleted and dLZ; aa1357-1594 deleted) used in immunoprecipitation and promoter assay. LZ, leucine zipper. (B) HA-tagged hSNF5 was cotransfected with the plasmids encoding flag-tagged RB1CC1wt or mutants in HEK293 cells. Anti-HA immunoprecipitated lysate and total cell lysate were analyzed by Western blotting. (C) Luciferase plasmid containing p16 promoter was transfected into HeLa cells together with RB1CClwt or mutants. Luciferase activity was calculated as the relative fold induction of each versus the value of co-transfection with pcDNA. The values indicated the means \pm standard errors from quadruplicate experiments in HeLa cells. Student's t-test; double-asterisks ( ${ }^{* *} \mathrm{p}<0.01$ ) indicate statistically significant differences between RB1CC1wt and the mutants.

inadequately activate the $p 16$ promoter, while RB1CC1wt or the dCCB mutant activated it adequately. In summary, $\mathrm{RB} 1 \mathrm{CC} 1$ could activate the $p 16$ promoter, depending to a large extent on the interaction with hSNF5.

\section{Discussion}

INK4a/CDKN2a/p16 functions as an inhibitor of D-type cyclin-dependent kinases (CDK4 and CDK6) that initiate phosphorylation of RB1, so p16 has the capacity to arrest cells in G1 phase of the cell cycle (1). Transcription of p16 is activated by Ets1/2 $(5,6)$ and hSNF5, a chromatin-remodeling factor (2-4). Reciprocally, BMI-1, a polycomb protein, and Id1, a bHLH protein, are known as negative regulators of p16 (7-9).

RB1CC1 enhances the transcription of RB1 (11), and forms a complex with hSNF5 and/or p53 (12). Nuclear RB1CC1 expression was correlated with a high p16 expression (Fig. 1A-B), so it was suggested that RB1CC1 activated the $p 16$ promoter. In the present study, hSNF5 was expressed in all of the breast cancer cases, and there was no correlation between hSNF5 and p16 expressions (Fig. 1C). Following-up on these immunohistochemical results; we analyzed the mechanism of how RB1CC1 controls p16 expression.

To detect the RB1CC1-activated site of the p16 promoter, we performed the promoter mapping using the luciferasereporter assay. A limited domain (-624 bp to $-22 \mathrm{bp}$ ) of the p16 promoter was required in a dose (DNA-length)-dependent manner for the transcriptional activation caused by RB1CC1 (Fig. 2A). We screened the $p 16$ promoter from -624 bp to -22 $\mathrm{bp}$, to identify the critical narrow domain of the promoter, but were unable to find it (Fig. 2B-C). These results made us hypothesize that another essential important factor might be a mediator, not in a sequence-specific but in a dose (DNA-length) -dependent manner, when the p16 transcription was activated by RB1CC1. Previously, we indicated that RB1CC1 interacted with hSNF5 (12) and suggested that $\mathrm{RB} 1 \mathrm{CC} 1$ activated the $p 16$ promoter through the interaction with hSNF5. 
To identify the possible cooperation between RB1CC1 and hSNF5 in activating the $p 16$ promoter, we evaluated whether RB1CC1 and hSNF5 bound to the promoter at -711 to $-529 \mathrm{bp}$. It was revealed that both molecules bound to this region of the $p 16$ promoter in HeLa cells, while neither could bind there in hSNF5-null TTC642 cells (Fig. 3). In addition, our preliminary ChIP-sequence data using the next-generation sequencer (Solid, Life Science Technology) suggested that RB1CC1 bound to the -621 to $-575 \mathrm{bp}$ region of the p16 promoter (data not shown) in HeLa cells. This region of the p16 promoter is consistent with the binding region of hSNF5 that has been reported previously $(3,4)$. Together, it is strongly suggested that hSNF5 functions as an essential interacting molecule when RB1CC1 binds to the 16 promoter. hSNF5 is a chromatin-remodeling factor that can activate the promoters without requiring specific binding sequences. Therefore, $\mathrm{RB} 1 \mathrm{CC} 1$ activated the $p 16$ promoter not in a sequencespecific, but in a dose (DNA-length)-dependent manner in our luciferase-reporter assay.

RB1CC1 binds to hSNF5 (12) as well as p53 (15), so the promoter enhancements caused by RB1CC1 were compared among HeLa, TTC642 (hSNF5-null) and H1299 (p53-null) cells, in order to validate the requirement for hSNF5 or p53 when RB1CC1 activated RB1, p16, p21 and TP53AIP1. It was revealed that $\mathrm{RB} 1 \mathrm{CC} 1$ required p53 as an essential molecule to activate $p 21$ and TP53AIPI, which are known as p53-targeting genes. Considering the $R B 1$ promoter, $\mathrm{RB} 1 \mathrm{CC} 1$ required hSNF5 rather than p53. Reciprocally, in order to activate 16 promoter, RB1CC1 required not only hSNF5 but also p53 as the important partners (Fig. 4). These results suggested that RB1CC1 might importantly form a large transcriptional complex with hSNF5 as well as p53 for p16 promoter enhancement.

Finally, we examined the binding between RB1CC1 and hSNF5 and the activation of $p 16$, because it had been reported that hSNF5 was the upstream transcriptional activator for $p 16$ (2-4). It was revealed that hSNF5 bound to the C-terminal region of $\mathrm{RB} 1 \mathrm{CC} 1$, and that an $\mathrm{RB} 1 \mathrm{CC} 1$ mutant without this region reduced significantly the ability to activate the pl6 promoter (Fig. 5). Together with the finding that the C-terminus of RB1CC1 is essential for its nuclear localization (11), it is suggested that RB1CC1 can bind to hSNF5 in cell nuclei and is recruited to the $p 16$ promoter by hSNF5 in order to activate the promoter. hSNF5 is expressed in most cancer cells, while hSNF5 is specifically deleted only in MRT, a childhood rare malignancy. In many different types of cancer, other factors, including RB1CC1, may play important roles in p16 expression, although hSNF5 can recruit them to the promoter region.

In conclusion, the present study revealed that RB1CC1 activates the 16 promoter and enhances p16 expression, and that hSNF5 is an essential interacting molecule that makes it possible for RB1CC1 to play this role. Further studies on RB1CC1 are needed in order to help clarify the way forward in research on oncogenesis and senescence. Clinically, the evaluation of RB1CC1 status is a possible biomarker for the prognostic indicator in patients with various types of cancer.

\section{Acknowledgements}

This study was supported in part by KAKENHI (Grant-in-Aid for Scientific Research on Priority Areas; No. 20012025 and 21592040) from the Ministry of Education, Culture, Sports, Science, and Technology, Japan; Japanese Society of Laboratory Medicine Fund for the Promotion of Scientific Research. These funding sources had no influence on the design of the study or in the collection, analysis, or interpretation of the data. The authors thank Hiroko Kita and Sawako Hirayama for experimental assistance; Ken-ichi Isobe for p21-luciferase plasmid; Hirofumi Arakawa for TP53AIP1luciferase plasmid. The authors declare that no competing interests exist.

\section{References}

1. Ohtani N, Yamakoshi K, Takahashi A and Hara E: The p16INK4a-RB pathway: molecular link between cellular senescence and tumor suppression. J Med Invest 51: 146-153, 2004.

2. Betz BL, Strobeck MW, Reisman DN, Knudsen ES and Weissman BE: Re-expression of hSNF5/INI1/BAF47 in pediatric tumor cells leads to G1 arrest associated with induction of p16ink4a and activation of RB. Oncogene 21: 5193-5203, 2002.

3. Oruetxebarria I, Venturini F, Kekarainen T, et al: P16INK4a is required for hSNF5 chromatin remodeler-induced cellular senescence in malignant rhabdoid tumor cells. J Biol Chem 279: 3807-3816, 2004.

4. Chai J, Charboneau AL, Betz BL and Weissman BE: Loss of the hSNF5 gene concomitantly inactivates p21CIP/WAF1 and p16INK4a activity associated with replicative senescence in A204 rhabdoid tumor cells. Cancer Res 65: 10192-10198, 2005.

5. Ohtani N, Zebedee Z, Huot TJ, et al: Opposing effects of Ets and Id proteins on p16INK4a expression during cellular senescence. Nature 409: 1067-1070, 2001.

6. Huot TJ, Rowe J, Harland M, et al: Biallelic mutations in p16(INK4a) confer resistance to Ras- and Ets-induced senescence in human diploid fibroblasts. Mol Cell Biol 22: 8135-8143, 2002.

7. Dhawan S, Tschen SI and Bhushan A: Bmi-1 regulates the Ink4a/Arf locus to control pancreatic beta-cell proliferation. Genes Dev 23: 906-911, 2009.

8. Alani RM, Young AZ and Shifflett CB: Id1 regulation of cellular senescence through transcriptional repression of p16/Ink4a. Proc Natl Acad Sci USA 98: 7812-7816, 2001.

9. Fan C, He L, Kapoor A, et al: Bmil promotes prostate tumorigenesis via inhibiting p16(INK4A) and p14(ARF) expression. Biochim Biophys Acta 1782: 642-648, 2008.

10. Kim WY and Sharpless NE: The regulation of INK4/ARF in cancer and aging. Cell 127: 265-275, 2006.

11. Ikebuchi K, Chano T, Ochi Y, et al: RB1CC1 activates the promoter and expression of RB1 in human cancer. Int $\mathrm{J}$ Cancer 125: 861-867, 2009.

12. Chano T, Ikebuchi K, Ochi Y, et al: RB1CC1 activates RB1 pathway and inhibits proliferation and cologenic survival in human cancer. PLoS One 5: e11404, 2010.

13. Chano T, Saji M, Inoue H, et al: Neuromuscular abundance of RB1CC1 contributes to the non-proliferating enlarged cell phenotype through both RB1 maintenance and TSC1 degradation. Int J Mol Med 18: 425-432, 2006.

14. Chano T, Ikegawa S, Kontani K, Okabe H, Baldini N and Saeki Y: Identification of RB1CC1, a novel human gene that can induce RB1 in various human cells. Oncogene 21: 1295-1298, 2002.

15. Melkoumian ZK, Peng X, Gan B, Wu X and Guan JL: Mechanism of cell cycle regulation by FIP200 in human breast cancer cells. Cancer Res 65: 6676-6684, 2005. 